\title{
Water partitioning between liquid iron and silicate melt
}

\author{
YUNGUO LI ${ }^{1 *}$, LIDUNKA VOČADLO ${ }^{1}$, TAO SUN $^{2}$, AND \\ JOHN P. BRODHOLT ${ }^{1,3}$ \\ ${ }^{1}$ Department of Earth Sciences, University College London, \\ Gower Street, London WC1E 6BT, United Kingdom \\ ${ }^{2}$ Key Laboratory of Computational Geodynamics, College of \\ Earth and Planetary Sciences, University of Chinese \\ Academy of Sciences, Beijing 100049, China \\ ${ }^{3}$ Centre for Earth Evolution and Dynamics, University of \\ Oslo, Oslo, Norway
}

The distribution of water in the Earth encodes key information for many Earth processes, and our understanding of the Earth and its history critically depends on the evolution of water in the Earth. Current estimates of the budget and distribution of water in the Earth have large uncertainties, most of which come from the lack of information about the deep Earth ${ }^{[1]}$.

We have calculated the partitioning of water between iron and silicate melts at 20-135 gigapascals and 2800-5000 kelvin, using $a b$ initio molecular dynamics and thermodynamic integration techniques. Our results indicate a siderophile nature of water at core-mantle differentiation and core-mantle boundary conditions, which can be weakened with increasing temperature; nevertheless, we find that water always partitions strongly into iron liquid at both reducing and oxidising conditions. The siderophile nature of water has also been verified by an empirical counting method showing the distribution of hydrogen in an equilibrated iron and silicate melt.

In light of accumulated evidence of early-Earth nebular gas ingasssing ${ }^{[2,3]}$, we therefore conclude that the Earth's core can act as a large reservoir containing most of the Earth's water. The findings can further constrain Earth accretion models and water distribution, and, importantly, hydrogen must be considered when attempting to determine the composition of the Earth's core.

[1] Peslier et al. (2017) Space. Sci. Rev. 212, 743-810. [2] Williams \& Mukhopadhyay (2019) Nature 565, 78-81. [3] Olson \& Sharp (2018) EPSL 498, 418-426. 